\title{
From Structural Biology to Small Molecule Therapy for Inborn Errors of Metabolism
}

Wyatt W. Yue

Structural Genomics Consortium, Nuffield Department of Medicine, University of Oxford

Inborn errors of metabolism (IEM) represents a group of 500 monogenic disorders each associated with inherited defect in a metabolic enzyme, regulatory protein, or complex. Despite the wellestablished genetic linkage and clinical manifestations for IEMs, there remains a lack of transformative therapy for many of them. This is in large part due to the conceptual challenge that the majority of IEMs result in the loss of enzyme function, rendering pharmacologic rescue difficult. Our team at the Structural Genomics Consortium (SGC), University of Oxford aims to address this gap using structural biology approaches to enable drug discovery for IEMs.

This lecture will present our recent highlights, citing examples from diverse pathways of carbohydrate, amino acid and vitamin metabolism, towards three key research objectives: (i) determination of novel protein structures to inform the biochemical defects of disease causing mutations, (ii) proof-of-concept studies to rationalize therapeutic approaches of substrate reduction and pharmacological chaperoning, and (iii) use of crystallography-based fragment screening to identify novel chemical matter and druggable pockets for small molecule development. 